\title{
The Effect of the New Eighth Edition Breast Cancer Staging System on 100 Consecutive Patients
}

\author{
Ashley Biswal $^{\mathrm{a}}$, Jacqueline Erler ${ }^{\mathrm{a}}$, Omar Qari ${ }^{\mathrm{a}}$, Arthur A. Topilow ${ }^{\mathrm{a}, \mathrm{b}}$, e, Varsha Gupta ${ }^{\mathrm{b}}$, \\ Mohammad A. Hossain ${ }^{\mathrm{b}}$, Arif Asif ${ }^{\mathrm{b}}$, Brian Erler ${ }^{\mathrm{c}}$, Denise Johnson Miller ${ }^{\mathrm{d}}$
}

\begin{abstract}
Background: In October 2016 the American Joint Committee on Cancer published the early eighth edition breast cancer prognostic staging system, incorporating biomarkers into previously accepted staging. The updated and current eighth edition became effective nationwide in January 2018 after a large update to its staging guidelines. This study's aim was to compare patients' anatomic seventh edition (anatomic), early eighth (pre-update, prognostic), and current eighth (post-update, prognostic) pathological stages and to assess the utility of recent inclusions to staging criteria. Additionally, we observed how the aforementioned stage changes aligned with breast cancer histologic subtypes.
\end{abstract}

Methods: An Institutional Review Board (IRB)-approved retrospective chart review was performed. Inclusion criteria included female patients between the ages of 35 to 95 years with a diagnosis of invasive ductal or lobular carcinoma of the breast $(n=100)$ at three Hackensack Meridian Health hospitals. The study evaluated any trends in patients' stage changes between the seventh edition, early eighth edition, and current eighth edition breast cancer staging guidelines. Breast cancer restaging was performed using a novel staging tool on Microsoft Excel.

Results: Only $26 \%$ of patients' stages changed when comparing the seventh edition stage vs. current eighth edition prognostic staging, most of which were downstaged. When comparing the seventh with early eighth edition prognostic staging, $38 \%$ of the patients' stages changed, with a majority of them being upstaged. Lastly, $95 \%$ of total stage changes were downstages between the early eighth and current eighth edition staging guidelines.

Conclusions: When comparing the seventh edition vs. current eighth

Manuscript submitted February 28, 2019, accepted April 5, 2019

${ }^{a}$ Office of Research Administration, Jersey Shore University Medical Center, 19 Davis Avenue, Neptune, NJ, USA

${ }^{b}$ Department of Medicine, Jersey Shore University Medical Center, 1945 Corlies Avenue, Neptune, NJ, USA

'Department of Pathology, Jersey Shore University Medical Center, 1945 Corlies Avenue, Neptune, NJ, USA

${ }^{\mathrm{d} D e p a r t m e n t}$ of Surgery, Jersey Shore University Medical Center, 1945 Corlies Avenue, Neptune, NJ, USA

${ }^{\mathrm{e}}$ Corresponding Author: Arthur A. Topilow, 31 Mahoras Drive, Ocean, NJ 07712, USA. Email: Arthur.Topilow@hackensackmeridian.org

doi: https://doi.org/10.14740/jocmr3803 edition staging, few patients (especially those with early stage cancer) underwent a stage change. However, there were significant changes in stage when comparing early eighth vs. current eighth stages. Considering these changes were mostly downstages and many patients reverted to their original seventh edition stage, the current eighth edition is based on a personalized, less radical staging approach, one that is more synonymous with original seventh edition staging.

Keywords: Breast cancer; AJCC; Clinical biomarkers; Histologic subtypes; Prognostic staging

\section{Introduction}

In the United States, approximately over 200,000 new cases of breast cancer are diagnosed yearly, and it is the second leading cause of cancer death among women [1,2]. Traditionally, the American Joint Committee on Cancer (AJCC) has recommended the usage of anatomical features, as seen in the seventh edition AJCC cancer staging manual, to classify breast cancer tumors. The TNM (tumor size $(\mathrm{T})$, nodal status $(\mathrm{N})$, metastases (M)) staging system categorizes the extent and location of a breast cancer tumor without needing clinical laboratory testing [3]. In October 2016, the AJCC published the eighth edition breast cancer prognostic staging system ("early eighth edition"). This incorporated the usage of biomarkers into previously accepted TNM staging [4]. This addition to staging guidelines recognizes the utility of several biologic markers such as estrogen receptor (ER), progesterone receptor (PR), human epidermal growth factor receptor-2 (HER2). Histological grades are also included in the eighth edition breast cancer staging system. Consequently, breast cancer patients are assigned a prognostic stage based on information gathered from TNM classification and the four aforementioned biomarkers. The AJCC eighth edition staging system integrates clinical laboratory testing for a more comprehensive and personalized prognostic staging criteria [5].

Following a critical update to its staging criteria, the AJCC eighth edition became effective in January 2018 ("current eighth edition"). The early eighth edition was reevaluated to account for "discrepancies" such as a single breast cancer staging system for in situ and invasive carcinomas that was separated into two different prognostic staging systems [6]. As a result, the Clinical Prognostic Stage Group (anatomic staging) and Pathological Prognostic Stage Group (anatomic + 
biomarker staging) were created. Also, criteria were added to the current eighth edition which better incorporates the usage of multigene panels for additional prognostic insight.

This study aims to provide insight for patients and physicians on how breast cancer prognostic stages changed between the anatomic seventh edition, early eighth edition (pre-update AJCC eighth edition), and current eighth edition (post-update AJCC eighth edition). It was of additional interest to evaluate how restaging affected the relationship between breast cancer's relative prognostic severities and its histologic subtypes: luminal A, luminal B, luminal B HER2 enriched, HER2 positive, and triple negative [7]. Patient restaging was done using a prognostic restaging tool designed to automatically provide a current AJCC eighth edition breast cancer stage. The study's aim is of clinical importance since restaged tumors are accompanied with potential modifications to treatment options. By upstaging a tumor for example, it will be categorized as more severe and will consequently require a more aggressive therapeutic approach. While there have been several large-scale foreign studies assessing these stage changes, this study analyzes the stage changes in a suburban American academic medical center [8-11].

\section{Materials and Methods}

\section{Selection and description of participants}

Upon receiving the Institutional Review Board approval, a retrospective chart review was conducted for 100 breast cancer patients from January 1, 2017 to May 30, 2018 at three hospitals within the Hackensack Meridian Health system: Jersey Shore University Medical Center, (JSUMC), Riverview Medical Center (RMC), and Ocean Medical Center (OMC). All clinical data were compiled from electronic medical records and obtained from the Meridian Tumor Registry. Inclusion criteria included female patients between the ages of 35 to 95 years with a diagnosis of invasive ductal or lobular carcinoma of the breast. Patient data included age, race, sex, and TNM information. To restage patients according to the early and current AJCC eighth edition staging system, biomarker status was recorded for ER, PR, HER2, as well as the Oncotype Dx score. Imaging results were collected from magnetic resonance imaging (MRI), computed tomography (CT), positron emission tomography (PET) and mammogram scans. Pathology reports were reviewed.

\section{Technical information and statistics}

Patients were restaged from the anatomic seventh edition to early eighth edition and current eighth edition stages using a prognostic staging tool programmed at JSUMC. The Microsoft Excel program provides a breast cancer prognostic stage according to the current eighth edition guidelines (Supplementary 1) (www. jocmr.org). Once information such as T, N, M status, histologic grade, biomarker statuses, and multigene panel results are entered into the program, a current eighth edition cancer stage (IA, IB, IIA, IIB, IIIA, IIIB, IIIIC, and IV) is generated. Additionally, descriptive statistics were collected to assess trends in patients'
Table 1. Demographic and Pathological Characteristics of Studied Breast Cancer Patients $(N=100)$

\begin{tabular}{|c|c|c|}
\hline Characteristic & $\mathbf{N}$ & $\%$ of cohort \\
\hline \multicolumn{3}{|l|}{ Age at diagnosis } \\
\hline $35-50$ & 12 & $12 \%$ \\
\hline$>50$ & 88 & $88 \%$ \\
\hline \multicolumn{3}{|l|}{ Ethnicity } \\
\hline White & 90 & $90 \%$ \\
\hline Black & 5 & $5 \%$ \\
\hline Asian Indian & 1 & $1 \%$ \\
\hline Other & 3 & $3 \%$ \\
\hline N/A & 1 & $1 \%$ \\
\hline \multicolumn{3}{|l|}{ Tumor size grade } \\
\hline $\mathrm{T} 1$ & 70 & $70 \%$ \\
\hline $\mathrm{T} 2$ & 24 & $24 \%$ \\
\hline $\mathrm{T} 3$ & 6 & $6 \%$ \\
\hline \multicolumn{3}{|l|}{ Nodal status grade } \\
\hline No & 79 & $79 \%$ \\
\hline N1 & 14 & $14 \%$ \\
\hline N1mi & 3 & $3 \%$ \\
\hline N2 & 3 & $3 \%$ \\
\hline N3 & 1 & $1 \%$ \\
\hline \multicolumn{3}{|l|}{ Metastases grade } \\
\hline M0 & 99 & $99 \%$ \\
\hline M1 & 1 & $1 \%$ \\
\hline \multicolumn{3}{|l|}{ Histological grade } \\
\hline G1 & 20 & $20 \%$ \\
\hline G2 & 52 & $52 \%$ \\
\hline G3 & 28 & $28 \%$ \\
\hline
\end{tabular}

aTumor size grade, nodal status grade, and metastases grade are used for anatomic seventh edition staging of breast cancer tumors.

stage changes between the seventh edition, early eighth edition, and current eighth edition. Observed stage changes between the three staging guidelines were arranged into various graphs.

\section{Data availability statement}

The datasets generated during and/or analyzed during the current study are available from the corresponding author on reasonable request.

\section{Results}

\section{Patient characteristics}

Among the 100 patients reviewed for this study, the mean age was 65.9 years old, with ages ranging from 35 - 95 (Table 1). A 
Table 2. Pathological Classification and Biomarker Status of Studied Breast Cancer Patients $(\mathrm{N}=100)$

\begin{tabular}{|c|c|c|}
\hline Characteristic & $\mathbf{N}$ & $\%$ of cohort \\
\hline \multicolumn{3}{|l|}{ Histology } \\
\hline Ductal & 82 & $82 \%$ \\
\hline Lobular & 16 & $16 \%$ \\
\hline Other & 2 & $2 \%$ \\
\hline \multicolumn{3}{|l|}{ ER status } \\
\hline Positive & 87 & $87 \%$ \\
\hline Negative & 13 & $13 \%$ \\
\hline \multicolumn{3}{|l|}{ PR status } \\
\hline Positive & 70 & $70 \%$ \\
\hline Negative & 30 & $30 \%$ \\
\hline \multicolumn{3}{|l|}{ HER2 status } \\
\hline Positive & 14 & $14 \%$ \\
\hline Negative & 86 & $86 \%$ \\
\hline \multicolumn{3}{|l|}{ Ki67 } \\
\hline Positive $(<10 \%)$ & 32 & $32 \%$ \\
\hline Intermediate (10-20\%) & 10 & $10 \%$ \\
\hline Negative $(\geq 20 \%)$ & 40 & $40 \%$ \\
\hline N/A & 18 & $18 \%$ \\
\hline \multicolumn{3}{|l|}{ Oncotype Dx score } \\
\hline Low-risk $(<18)$ & 25 & $25 \%$ \\
\hline Intermediate (18 - 30) & 15 & $15 \%$ \\
\hline High-risk $(\geq 31)$ & 1 & $1 \%$ \\
\hline Test not performed & 42 & $42 \%$ \\
\hline N/A & 17 & $17 \%$ \\
\hline
\end{tabular}

ER: estrogen receptor; PR: progesterone receptor; HER2: human epidermal growth factor receptor-2. majority of the cases were Caucasian patients and about $5 \%$ of patients were African American. In order to understand stage changes between the seventh edition and the early and current AJCC eighth edition staging systems, a variety of histological grades were observed. A majority of tumor histology from hospital pathology analysis showed that $82 \%$ of cases were ductal carcinomas while $16 \%$ of cases were lobular carcinomas.

An analysis of biomarker status was also included in the study (Table 2). In regards to the ER and PR most cases were positive. Approximately $86 \%$ of cases were HER2 negative. The Ki-67 protein test, a cellular marker for proliferation, is a laboratory test used to measure the extent of a tumor. Thirtytwo percent of patients tested positive for the Ki67 protein test and $40 \%$ of patients tested negative. When recording the Oncotype Dx score, a multigene panel test, $25 \%$ were categorized as "low risk" for tumor recurrence.

\section{Comparison of breast cancer stages}

There were several evident changes when assessing stage distribution for the anatomic seventh edition, early eighth edition, and current eighth edition (Fig. 1). Stage distribution according to the seventh edition anatomic staging was: 64\% patients stage IA, 20\% stage IIA, $8 \%$ stage IIB, 6\% stage IIIA, 1\% stage IIIC, and $1 \%$ stage IV. Observed breast cancer cases staged for the early eighth edition system demonstrated that $57 \%$ patients were classified as stage IA, $16 \%$ as stage IB, $6 \%$ as stage IIA, $7 \%$ as stage IIB, $6 \%$ as stage IIIA, $5 \%$ as stage IIIB, $2 \%$ as stage IIIC, and $1 \%$ of observed cases as stage IV. Lastly, for the current eighth edition, stage distribution was: $70 \%$ patients stage IA, $12 \%$ stage IB, $7 \%$ stage IIA, 4\% stage IIB, $5 \%$ stage IIIA, $1 \%$ stage IIIC, and $1 \%$ stage IV.

Notably, there were no IB staged cases in the seventh edition criteria. When patients were restaged to reflect the eighth edition's guidelines, the population saw a $16 \%$ increase in

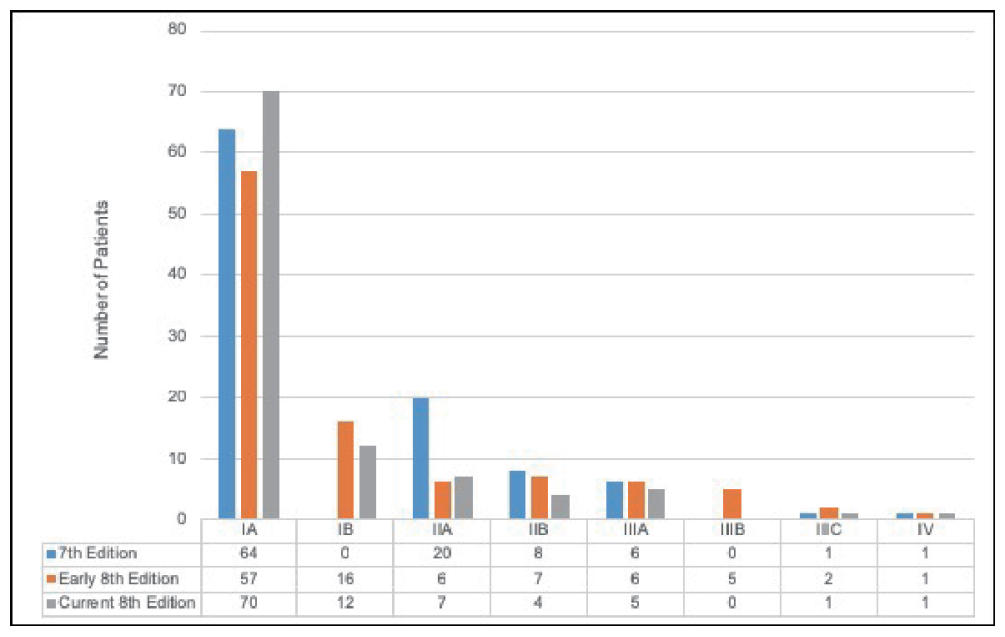

Figure 1. Distribution of the seventh edition anatomic stage, early eighth edition prognostic stage, and current eighth edition prognostic stage among breast cancer patients $(n=100)$. 


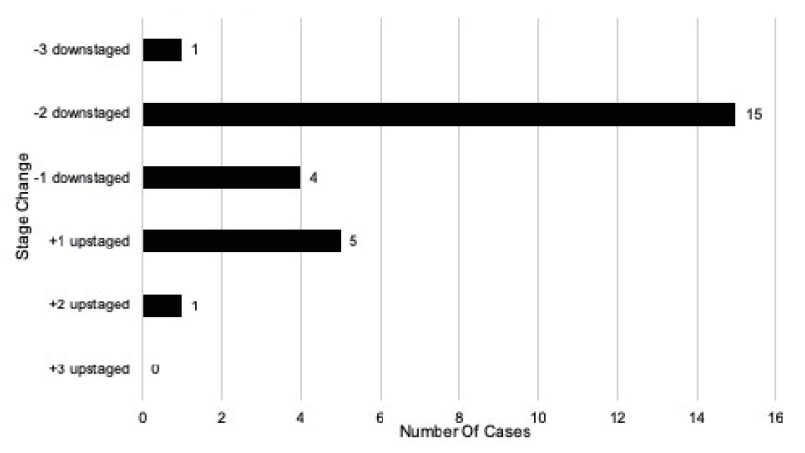

Figure 2. Stage changes in the seventh edition vs. current eighth edition of breast cancer prognostic stage $(n=100)$.

IB cases for the early eighth edition and a $12 \%$ increase in IB cases for the current eighth edition. For IIA staged cases, the early and current eighth editions show a decrease in cases as compared to the seventh edition anatomic staging system. Additionally, IA tumors were consistently the most common across all three staging criteria.

\section{The seventh vs early eighth and current eighth edition breast cancer staging}

When comparing stage changes between the anatomic seventh and current eighth edition prognostic staging systems, 26 $(26 \%)$ of total cases underwent a stage change (Fig. 2); and $23.1 \%(6 / 26)$ of those cases were upstages. Out of the 26 total stage changes, five cases $(19.2 \%)$ were upstaged by one level and one case $(3.8 \%)$ upstaged by two levels. There was no case that upstaged by three levels. ) Of the total stage changes, $76.9 \%(20 / 26)$ of them were downstages. Out of the 26 total stage changes, four cases $(15.4 \%)$ were downstaged by one level, $15(57.7 \%)$ downstaged by two levels, and one case (3.8\%) downstaged by three levels. A comparison was also performed with patients' stage changes between the anatomic seventh and early eighth edition (Fig. 3).

There were several changes when comparing the anatomic seventh edition stages with the current eighth edition prognostic staging system. Out of the 64 patients with stage IA according to the seventh edition, $60(93.8 \%)$ remained prognostic stage IA, and four cases $(6.3 \%)$ were upstaged to prognostic

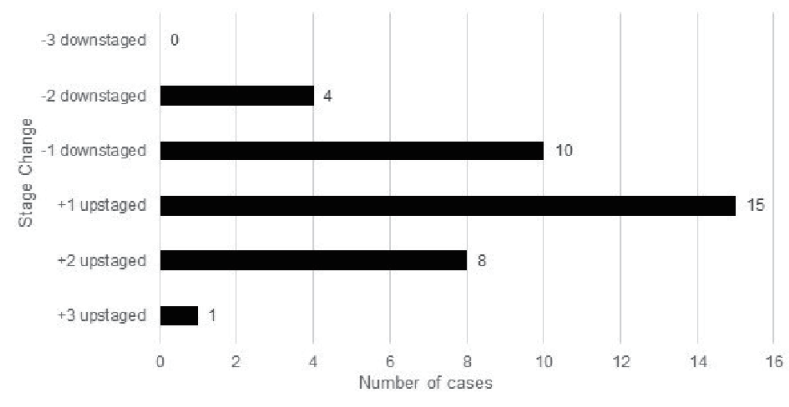

Figure 3. Stage changes in the seventh edition vs. early eighth edition of breast cancer prognostic stage $(n=100)$.

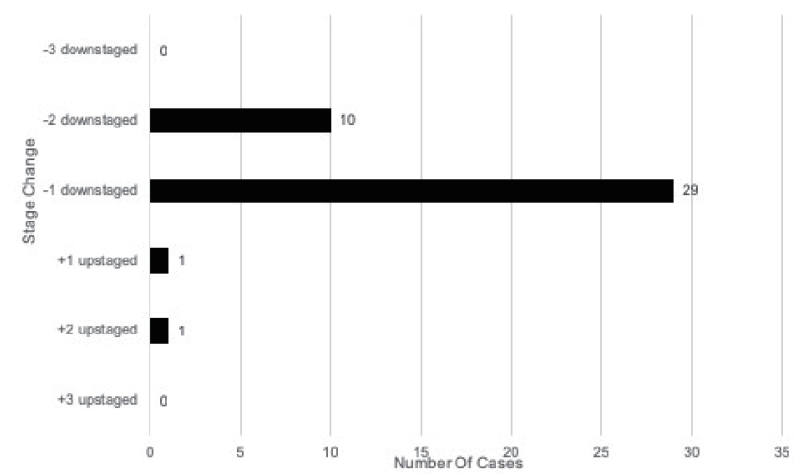

Figure 4. Stage changes in early eighth edition vs. current eighth edition of breast cancer prognostic stage $(n=100)$.

stage IB. Out of the 20 patients with stage IIA according to the seventh edition, seven $(35.0 \%)$ remained prognostic stage IIA, $10(50.0 \%)$ downstaged to prognostic stage IA, and three $(15.0 \%)$ downstaged to prognostic stage IB. There were eight patients with stage IIB according to the anatomic seventh edition, three $(37.5 \%)$ remained prognostic stage IIB, four $(50.0 \%)$ downstaged to prognostic stage IB, and one $(12.5 \%)$ upstaged to prognostic stage IIIA. Out of six anatomic stage IIIA patients, three $(50.0 \%)$ remained prognostic stage IIIA, one $(16.7 \%)$ downstaged to prognostic stage IB, one $(16.7 \%)$ downstaged to prognostic stage IIB, and one (16.7\%) upstaged to prognostic stage IIIC. Only one patient $(100 \%)$ with stage IIIC according to the anatomic seventh edition was downstaged to prognostic stage IIIA. Lastly, one patient (100\%) with stage IV according to the anatomic seventh edition remained prognostic stage IV.

The early eighth edition vs. current eighth edition breast cancer staging systems

When comparing stage changes between the early prognostic and current prognostic eighth edition staging systems, 41 $(41 \%)$ of total cases underwent a stage change (Fig. 4). Two $(2 / 41,4.9 \%)$ of the total stage changes were upstages. Out of the 41 total stage changes, one case $(2.4 \%)$ was upstaged by one level and one $(2.4 \%)$ upstaged by two levels. There was no case that was upstaged by three levels. Thirty-nine (39/41, $95.1 \%$ ) of the total stage changes were downstages. Out of the 41 total stage changes, $29(70.7 \%)$ were downstaged by one level and 10 (24.4\%) downstaged by two levels. There was no case that was downstaged by three levels.

There were several changes when comparing early eighth edition prognostic stage with the current eighth edition prognostic stage. Out of the 57 patients with early eighth edition prognostic stage IA, $55(96.5 \%)$ remained prognostic stage IA, one $(1.8 \%)$ upstaged to current eighth edition prognostic stage IB, and one (1.8\%) upstaged to current eighth edition prognostic stage IIA. Out of the 16 patients with early eighth edition prognostic stage IB, one $(6.3 \%)$ remained current eighth edition prognostic stage IB and 15 (93.8\%) downstaged to current eighth edition prognostic stage IA. Out of 

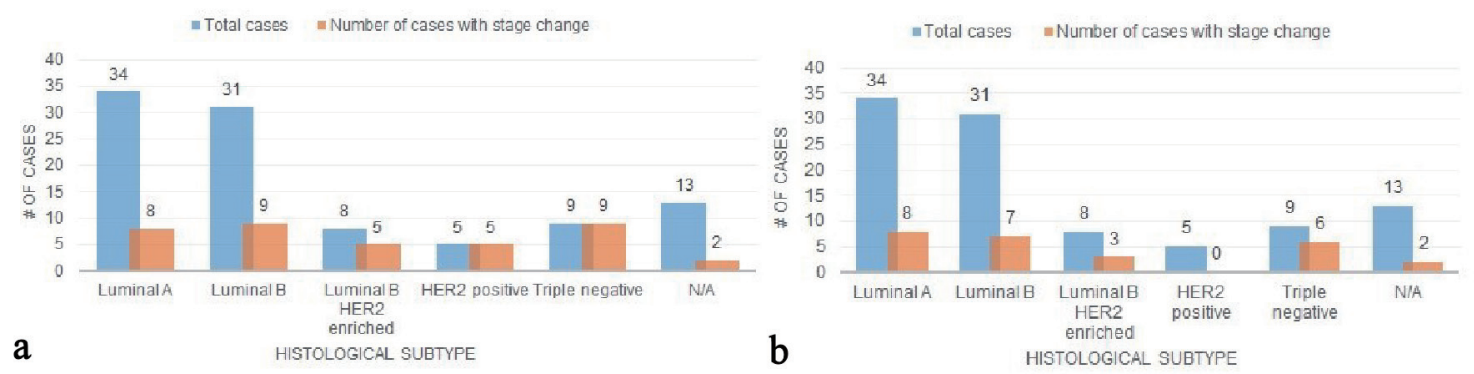

Figure 5. (a) Stage changes across histologic subtypes (the seventh vs. early eighth edition). (b) Stage changes across histologic subtypes (the seventh vs. current eighth edition).

six patients with early eighth edition prognostic stage IIA, six $(100 \%)$ downstaged to current eighth edition prognostic stage IB. Of the seven patients with early eighth edition prognostic stage IIB, four $(57.1 \%)$ downstaged to current eighth edition prognostic stage IB and three $(42.9 \%)$ downstaged to current eighth edition prognostic stage IIA. Out of six patients with early eighth edition prognostic stage IIIA, one $(16.7 \%)$ remained current eighth edition prognostic stage IIIA, three $(50.0 \%)$ downstaged to current eighth edition prognostic stage IIA, and two (33.3\%) downstaged to prognostic stage IIB. Out of five patients with early prognostic stage IIIB, two $(40.0 \%)$ downstaged to current eighth edition prognostic stage IIB and three $(60.0 \%)$ downstaged to prognostic stage IIIA. Out of two patients with early eighth edition prognostic stage IIIC, one $(50.0 \%)$ remained current eighth edition prognostic stage IIIC and one $(50.0 \%)$ downstaged to prognostic stage IIIA. Lastly, one $(100 \%)$ early eighth edition prognostic stage IV patient remained current prognostic stage IV.

\section{Breast cancer staging systems vs. histologic subtypes}

Out of the total population, the numbers of patients with luminal A, luminal B, luminal B HER2-enriched, HER2 positive, and triple negative histologic subtypes were: 34 (34.0\%), $31(31.0 \%), 8(8.0 \%), 5(5.0 \%), 9(9.0 \%)$. When comparing the seventh edition and early eighth edition prognostic stages, 23.5\% (8/34) luminal A patients, 29.0\% (9/31) luminal B patients, 62.5\% (5/8) luminal B HER-2 enriched patients, $100 \%$ $(5 / 5)$ HER2 positive patients, and $100 \%(9 / 9)$ triple negative patients underwent a stage change (Fig. 5a). A majority of the stage changes in luminal A and luminal B were downstages: $62.5 \%(5 / 8)$ and 55.6\% (5/9), respectively; and 100\% (5/5, 9/9) of HER2 and triple negative stage changes were upstages. When comparing the seventh edition and current eighth edition prognostic stages, 23.5\% (8/34) luminal A patients, $22.6 \%$ (7/31) luminal B patients, 37.5\% (3/8) luminal B HER-2 enriched patients, $0 \%(0 / 5)$ HER2 positive patients, and $66.7 \%$ (6/9) triple negative patients underwent a stage change (Fig. $5 b)$. One hundred percent $(8 / 8,7 / 7,3 / 3)$ of the luminal $A$, luminal B, and luminal B HER2 enriched stage changes were downstages; and $100 \%(6 / 6)$ of the triple negative stage changes were upstages.

In the current eighth edition staging, the distribution of histological subtypes was quite broad (Fig. 6). The majority of luminal A cases (82.4\%) were classified as prognostic stage IA. Similarly, the majority of luminal B cases $(80.6 \%)$ and HER2 enriched cases $(62.5 \%)$ were classified as prognostic stage IA. There were no triple negative cases that were classified as prognostic stage IA. A majority of triple negatives were classified as prognostic stages IB (44.4\%) and IIA $(33.3 \%)$.

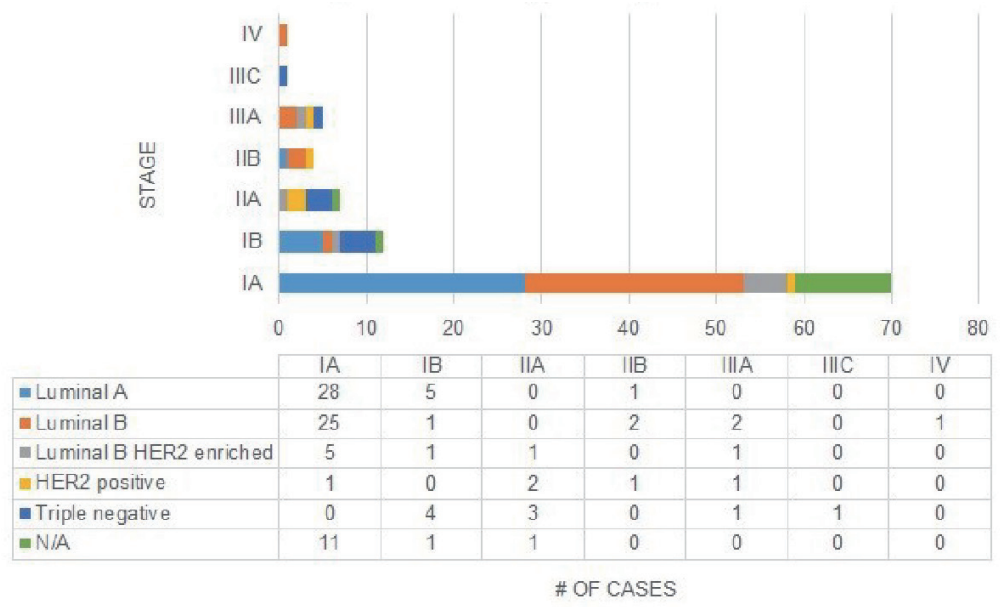

Figure 6. Distribution of stages across histologic subtypes in current eighth edition. 


\section{Discussion}

The AJCC's seventh edition cancer staging system was rooted in anatomic classification and afforded clinicians the ability to stage tumors without clinical laboratory tests [8]. When comparing the anatomic seventh vs. early eighth prognostic staging, few patients (especially those with early stage cancer) underwent a stage change (Fig. 3) (Supplementary 2) (www. jocmr.org). The same was observed for the anatomic seventh vs. current eighth prognostic staging. However, there were considerable changes in stage when comparing early eighth vs. current eighth edition prognostic stages. It was noted these changes were mostly downstages and many patients kept their original seventh edition stage after the update. The current eighth edition is based on a personalized, less radical staging approach, one that is more synonymous with original seventh edition staging. In the review of histologic subtypes, the staging changes are mixed (Figs. 5a, b and 6). Most luminal A and $B$ patients are stage IA and downstaged, and slightly more than half of the HER2 positive cases were classified as prognostic stage IA in the current eighth edition. Triple negative patients were upstaged in every observed instance. As a result, it is important to recognize the implications staging changes have on the patient [9].

By incorporating contemporary biomarkers into the previously accepted anatomic staging, the AJCC aims to provide more personalized staging and consequently, more personalized treatment options. It is an elaborate system based on history, physical examination, imaging studies, and relevant biopsies. It incorporates prior TNM criteria to tumor grade, HER2, $\mathrm{ER}$, and PR status. It also takes into account the recurrence score, the result of a multigene assay, called Oncotype Dx, for patients with hormone receptor-positive, HER2-negative, node-negative tumors that are $<5 \mathrm{~cm}$. It is important to note that the incorporation of contemporary biomarkers into treatment decisions has been done by clinicians prior to the AJCC publishing the early eighth edition staging manual.

In determining the long-term effects on the updated stage changes among breast cancer patients, a study would need to follow patients for an extended period of time. Given that all patients are alive in this study, it became difficult to detect the long-term effects of stage changes. The limitation of incorporating the current eighth edition staging system is that it is somewhat more complex than the prior TNM staging and its utilization will encourage additional laboratory genomic studies. There is a need for further research on breast cancer management employing the new staging system. This especially applies to developing countries considering that full breast cancer therapy is often cost prohibitive [10]. The challenge for research is to simplify the prognostic studies further while improving and refining prognostic prediction. In the bigger picture, these genomic and HER-2 studies could reduce the overall cost of care by improving outcomes and selecting patients who truly need extra treatment [11].

In the future, greater attention will likely be channeled towards genomic testing in order to predict the behavior of a tumor and responsiveness to drugs. The MammaPrint test, a genomic test made by Agendia, and the Prosigna Breast Can- cer Prognostic Gene Signature Assay by NanoString assess the activity of certain genes in early-stage breast cancer by classifying patients as either low risk or high risk of recurrence $[12,13]$. The MammaPrint test and the Prosigna assay analyze the activity of approximately 70 and 58 genes respectively by measuring its expression levels. This offers insight on how the tumor will eventually grow and spread [14]. Another genomic test, the Blueprint genomic lab test, is used in conjunction with the MammaPrint test to analyze the mutations that are dictating the tumor's behavior [15]. It allows clinicians to narrow tumor subtype into three classifications: luminal-type, HER2type, and basal-type. By combining this test's results with the MammaPrint test's recurrence risk, clinicians may be better equipped to navigate the aggressive treatment strategies involving early-stage breast cancer patients.

The Oncotype DX test has been readily incorporated into existing breast cancer staging guidelines and treatment decisions [16]. It is widely recognized in determining risk of recurrence in early-stage estrogen receptor positive breast cancer and has been tested in several large-scale studies such as the TAILORx trial [17]. Additionally, the Oncotype DX test relies on the activity of 21 genes to infer potential responsiveness to chemotherapy and radiotherapy (for cases involving ductal carcinoma in situ). The test provides a recurrence score which serves to guide physicians from a prognostic standpoint as well as a predictive one for responsiveness to certain treatment options [18]. The aforementioned commercially available tests show potential to gain widespread use. Clinicians are already using it to inform their treatment decisions involving chemotherapy and other therapeutic approaches.

Breast cancer knowledge is advancing steadily, especially after addition of biologic markers [19]. Use of these markers along with histologic grading is associated with improved survival independent of TNM staging [20]. Incorporation of these additional criteria to the prior TNM staging would therefore provide more precise and accurate staging, and is expected to improve overall patient outcomes through more precise treatment strategies [21]. Since biologic markers have directly been associated with selection of treatment strategies including decisions for chemotherapy and endocrine therapy, the new staging is expected to have a favorable impact on risk of recurrence, which will in turn be very important for the internist and primary care physician following the patient. It further implies that the new staging system will decrease the financial burden of cancer treatment since we know that the treatment of breast cancer becomes more expensive as the stage advances, owing to the fact that advance stage patients require more resource utilization [22].

\section{Conclusions}

With the introduction of the AJCC's eighth edition breast cancer staging system, there has been wide acknowledgement of the value of histochemical and genomic findings in the evaluation of breast cancer patients and treatment decisions. The inclusions made to the eighth edition staging system for breast cancer demonstrate the effort towards more precise and personalized treatment planning. A more detailed and scien- 
tific approach to classification of breast cancer patients such as the eighth edition will allow valid outcome comparisons to be made among various local and international study groups. Moving forward, the histologic subtype of a breast cancer patient remains important factors in determining a course of treatment for a patient. At this point, following the seventh and early eighth edition staging, we welcome the current eighth edition breast cancer staging system but acknowledge that with new scientific discoveries, it is bound to change again.

Majority of the patients included in this study are at an early breast cancer stage. The most evident change in stage distribution is that the current eighth edition has a greater amount of stage I patients than the anatomic seventh edition and early eighth edition. Implementation of the AJCC eighth edition staging system began in January 2018. Our research highlights that there is a need for further studies to assess the direct effect of the new staging system on overall survival, quality of life for the patient, and potential side effects due to changes in treatment. It would also help us make further revisions to the system in the setting of ever evolving multigene assays.

\section{Acknowledgments}

The authors wish to acknowledge JSUMC's Senior Tumor Registrar, Debbie Feeney, Mila Lachica, Research Coordinator, and the Office of Research Administration for providing technical support. Ashley Biswal and Jacqueline Erler were pre-medical student interns supported by a generous grant from the late Dr. Herbert R. Axelrod of Neptune, New Jersey and Zurich, Switzerland.

\section{Financial Disclosure}

This study was supported by an unrestricted research grant from the late Dr. Herbert R. Axelrod to Jersey Shore University Medical Center.

\section{Conflict of Interest}

The authors declare that there is no conflict of interest regarding the publication of this paper.

\section{Informed Consent}

The data for this study were solely obtained through a retrospective chart review. As a result, informed consent was not applicable.

\section{Author Contributions}

All of the authors listed contributed significantly to the research question, data collection, and writing of this manuscript.

\section{References}

1. Siegel RL, Miller KD, Jemal A. Cancer statistics, 2018. CA Cancer J Clin. 2018;68(1):7-30.

2. The National Breast Cancer Foundation. Breast Cancer Facts. Available at: https://www.nationalbreastcancer. org/breast-cancer-facts. Accessed January 3, 2019.

3. Giuliano AE, Edge SB, Hortobagyi GN. Eighth Edition of the AJCC Cancer Staging Manual: Breast Cancer. Ann Surg Oncol. 2018;25(7):1783-1785.

4. Hortobagyi GN, Connolly JL, D'Orsi CJ, et al. Breast. AJCC cancer staging manual, eighth edition. American Joint Committee on Cancer. 2018:589-632.

5. Amin MB, Greene FL, Edge SB, Compton CC, Gershenwald JE, Brookland RK, Meyer L, et al. The Eighth Edition AJCC Cancer Staging Manual: Continuing to build a bridge from a population-based to a more "personalized" approach to cancer staging. CA Cancer J Clin. 2017;67(2):93-99.

6. Giuliano AE, Connolly JL, Edge SB, Mittendorf EA, Rugo HS, Solin LJ, Weaver DL, et al. Breast cancer-major changes in the American Joint Committee on Cancer eighth edition cancer staging manual. CA Cancer J Clin. 2017;67(4):290-303.

7. Prat A, Pineda E, Adamo B, Galvan P, Fernandez A, Gaba L, Diez M, et al. Clinical implications of the intrinsic molecular subtypes of breast cancer. Breast. 2015;24(Suppl 2):S26-35.

8. Edge SB, Compton CC. The American Joint Committee on Cancer: the 7th edition of the AJCC cancer staging manual and the future of TNM. Ann Surg Oncol. 2010;17(6):1471-1474.

9. Zhou B, Xu L, Ye J, Xin L, Duan X, Liu Y. The prognostic value of the 8th edition of the American Joint Committee on Cancer (AJCC) staging system in HER2-enriched subtype breast cancer, a retrospective analysis. Anticancer Res. 2017;37(8):4615-4621.

10. National Comprehensive Cancer Network. NCCN framework for resource stratification of NCCN guidelines: invasive breast cancer. Available at: https://www.nccn.org/ professionals/physician_gls/pdf/breast_basic.pdf. Accessed January 28, 2019.

11. Abdel-Rahman O. Validation of the 8th AJCC prognostic staging system for breast cancer in a population-based setting. Breast Cancer Res Treat. 2018;168(1):269-275.

12. Brandao M, Ponde N, Piccart-Gebhart M. Mammaprint: a comprehensive review. Future Oncol. 2019;15(2):207224.

13. Alvarado MD, Prasad C, Rothney M, Cherbavaz DB, Sing AP, Baehner FL, Svedman C, et al. A prospective comparison of the 21-gene recurrence score and the PAM50Based Prosigna in estrogen receptor-positive early-stage breast cancer. Adv Ther. 2015;32(12):1237-1247.

14. Beumer IJ, Persoon M, Witteveen A, Dreezen C, Chin SF, Sammut SJ, Snel M, et al. Prognostic value of MammaPrint ((R)) in invasive lobular breast cancer. Biomark Insights. 2016;11:139-146.

15. Wuerstlein R, Kates R, Gluz O, Grischke EM, Schem 
C, Thill M, Hasmueller S, et al. Strong impact of MammaPrint and BluePrint on treatment decisions in luminal early breast cancer: results of the WSG-PRIMe study. Breast Cancer Res Treat. 2019.

16. Carlson JJ, Roth JA. The impact of the Oncotype Dx breast cancer assay in clinical practice: a systematic review and meta-analysis. Breast Cancer Res Treat. 2013;141(1):1322.

17. Sparano JA, Gray RJ, Makower DF, Pritchard KI, Albain KS, Hayes DF, Geyer CE, Jr., et al. Adjuvant chemotherapy guided by a 21 -gene expression assay in breast cancer. N Engl J Med. 2018;379(2):111-121.

18. National Public Radio (NPR). For some breast cancer patients, the chemo decision just got easier. Available at: www. npr.org/sections/health-shots/2018/06/03/616298863/forsome-breast-cancer-patients-the-chemo-decision-justgot-easier. Accessed January 3, 2019.
19. Williams BA, Chagin KM, Bash LD, Boden WE, Duval S, Fowkes FGR, Mahaffey KW, et al. External validation of the TIMI risk score for secondary cardiovascular events among patients with recent myocardial infarction. Atherosclerosis. 2018;272:80-86.

20. Krop I, Ismaila N, Stearns V. Use of biomarkers to guide decisions on adjuvant systemic therapy for women with early-stage invasive breast cancer: American society of clinical oncology clinical practice focused update guideline summary. J Oncol Pract. 2017;13(11):763-766.

21. Haque R, Xu X, Shi J, Kwan ML, Chlebowski RT. Breast cancer outcomes in a racially and ethnically diverse cohort of insured women. Ethn Dis. 2018;28(4):565-574.

22. Valencia OM, Samuel SE, Viscusi RK, Riall TS, Neumayer LA, Aziz H. The role of genetic testing in patients with breast cancer: a review. JAMA Surg. 2017;152(6):589594. 